\title{
Synchronization signal distortion in quantum communication systems
}

\author{
V.D. Dubrovskaia, S. A. Chivilikhin \\ ITMO University, Kronverkskiy, 49, St. Petersburg, 197101, Russia \\ vddubrovskaia@gmail.com
}

PACS 03.67.-a, 41.20.Jb

DOI 10.17586/2220-8054-2017-8-3-382-385

\begin{abstract}
An important problem in the practical implementation of fiber optical quantum communication systems is to synchronize the sender and receiver modules using a separate optical channel. The signal visibility in the quantum channel, which contributes to quantum bit error rate, is influenced by the synchronization signal delay. In this work, we investigate the dependence of the synchronization signal parameters on the dispersive effects in the fiber for a subcarrier wave quantum communication system (SCWQC), which is promising for quantum networking applications. The ITU-T G.652D standard single mode optical fiber was used for modeling. The maximum calculated phase mismatch of the synchronization signal for the system operating at $100 \mathrm{~km}$ fiber length corresponds to $1.7 \mathrm{ps}$ signal time delay. The results show that dispersion causes significant signal distortion, therefore additional phase adjustment at least every 2.3 hours is required for stable system operation.
\end{abstract}

Keywords: quantum communications, clock synchronization, temperature dependence of the signal, chromatic dispersion.

Received: 4 February 2017

Revised: 16 March 2017

Final revision: 28 May 2017

\section{Introduction}

The development of quantum technologies is opening up new potential applications in the use of quantum algorithms, creation and modeling of complex physical, biological systems, new physical methods of sending, receiving and processing information. To continue progress in these areas we need to evaluate the possibility of integrating quantum technologies and especially quantum communication (QC) with existing communication networks $[1,2]$.

A common approach to establishing long-distance links in QC is based on using optical signals transmitted through fibers. In this paper, we consider a subcarrier wave quantum communication system (SCWQC), important for practical applications [3]. One of the key features of the practical QC systems is the signal transmitted through a separate channel which synchronizes its transmitter and receiver modules. For errorless operation of the system, compensation mechanisms against negative effects of signal distortion should be considered $[4,5]$.

The synchronization signal time delay may occur as a result of external influences, and one of these main effects is temperature [6]. A temperature model of an optical cable was created earlier to consider real-life environment operating conditions of the SCWQC system [7]. Using it, in [7] we discussed refractive index dependence on temperature, which led to the signal delay, and the results showed that phase adjustment is required every $158 \mathrm{~ms}$ for stable system operation.

This paper investigates another signal factor which can contribute to synchronizing signal delay in optical fiber: using created temperature model $-\mathrm{s}$ distortion dependence of dispersion effects. We estimated the influence of daily temperature on signal temporal delay due to chromatic dispersion and apply the calculations for system improvement.

\section{Process modeling}

In a subcarrier wave quantum communication system phase matching between the transmitter and the receiver is defined by clock signals from respective voltage controlled oscillators. Thereby, the problem of system synchronization is reduced to fine tuning of the transmitter and receiver oscillators. Detailed descriptions of phase modulating in a SCWQC system are provided in $[8,9]$.

The main characteristics of the QC systems are determined by the QBER. This is defined as the ratio between the erroneous bits and the total number of received quantum bits [1]. Two main factors contribute to the QBER value: the signal visibility in the quantum channel and the dark count of the detector. Since the visibility value represents the probability of photon out-of-phase detection, it depends on the quality of optical phase matching of the transmitter and receiver phase modulators used for encoding qubits. For modern practical QC systems, the 
desired visibility value is usually around 98-99\%. As shown in [7], the minimum acceptable visibility value in SCWQC system is corresponding to maximum modulating signal phase mismatch $\Delta \phi=0.043$.

The relation between the phase and time delays $\Delta t$ of a signal with frequency $\Omega$ is given by:

$$
\Delta t=\frac{\Delta \phi}{\Omega}
$$

The $\Omega$ value is typically several gigahertz for SCWQC systems [3,8]; we used a value of $4 \mathrm{GHz}$ in our calculations. Therefore, the maximum tolerable synchronization signal time delay is $1.7 \mathrm{ps}$.

The temperature model developed in [7] is intended to account for the effect of cable heating on the occurrence optical signal delay. This model was created considering a typical daily temperature profile and parameters of a conventional optical cable, with ITU-T. G.652D standard single mode optical fibers deployed inside. The heat equation with new boundary conditions were solved to account for wind speed.

The two-dimensional heat equation for the cable is:

$$
\mathrm{c} \rho \frac{\partial T}{\partial t}=\frac{\partial}{\partial x}\left(\lambda \frac{\partial T}{\partial x}\right)+\frac{\partial}{\partial y}\left(\lambda \frac{\partial T}{\partial y}\right),
$$

where $\lambda$ is thermal conductivity, $\rho$ represents density and $c$ is heat capacity. New boundaries conditions are:

$$
\left.\lambda \frac{\partial T}{\partial r}\right|_{r=R}=\alpha(t)\left(T a(t)-\left.T\right|_{r=R}\right),
$$

where $r$ and $R$ are the radial coordinate and radius of the cable. The coefficient of convective heat exchange $\alpha$ depends on Nusselt number $N u$ and cable diameter $D[10]$ :

$$
\alpha=\lambda \cdot N u / D \text {, }
$$

Nusselt number depends on experimental coefficients $C$ and $m$ and Reynolds number Re:

$$
N u=C \cdot \operatorname{Re}^{m} \text {. }
$$

The Reynolds number is calculated on wind speed $V$ and kinematic viscosity $\eta$ :

$$
\operatorname{Re}=D V / \eta
$$

The wind speed changes periodically depending on time $V=V_{0}+V \cos (\omega t)$.

For fibers in the cable, the temperature change will decay with increasing distance from the cable outer jacket into the core of the fibers [11]:

$$
T=T_{0}+\delta T \exp \left(-\frac{a \tau}{h^{2}}\right) \cos (a t),
$$

where $T_{0}$ is the average daily temperature, $\delta T$ is the temperature variation, $h$ is the distance between cable outer jacket and the fiber core, $a$ is thermal diffusivity, $\tau$ is the time in seconds. The initial (mean) environmental temperature $T$ is chosen to be 15 degrees, with $\delta T=10$ degrees oscillation amplitude over a 24 hour period.

In the following section, we find the temperature dependence of synchronization signal phase using the created temperature model and numerically solve equations to find the dispersion contribution to synchronization signal delay.

\section{Chromatic dispersion temperature dependence}

To analyze the effect of the dispersion on the synchronization signal we consider total chromatic dispersion described by the third-order Sellmeier equation [12]. For the chosen optical fiber it can be modeled as:

$$
D(\lambda)=\frac{S_{0}}{4}\left(\lambda-\frac{\lambda_{0}^{4}}{\lambda^{3}}\right),
$$

where $\lambda_{0}$ is the zero dispersion wavelength, $\mathrm{S}_{0}$ is the chromatic dispersion slope at $\lambda$. Assuming both parameters depend on temperature, we then take the derivative the equation (2) with respect to temperature:

$$
\frac{d D}{d T}=\frac{1}{4}\left(\lambda-\frac{\lambda_{0}^{4}}{\lambda^{3}}\right) \frac{d S_{0}}{d T}-\frac{S_{0} \lambda_{0}^{3}}{\lambda^{3}} \frac{d \lambda_{0}}{d T} .
$$

Both $\frac{d S_{0}}{d T}$ and $\frac{d \lambda_{0}}{d T}$ contribute significantly to $\frac{d D}{d T}$ at a given wavelength. This component affects the signal distribution and may lead to incorrect operation of the QC system. To prevent this, it is necessary to take this effect into account. Fiber standard dispersion coefficient $D\left(T_{0}\right)$ is already accounted for in system operation. However, deviation from the standard value due to temperature can strongly affect the signal delay. Thus the second component should be considered as well. 
The total signal delay due to temperature effect is:

$$
\Delta t=\frac{d D}{d T}\left(T-T_{0}\right) \Delta \lambda L,
$$

where $L$ is the length of the cable, $\Delta \boldsymbol{\lambda}$ is the spectral width.

\section{Results and discussion}

We implemented temperature model used previously to determine the synchronization signal temporal delay due to chromatic dispersion in the SCWQC system with a cable length of $100 \mathrm{~km}$. The created model takes into account the size and thermal conductivity of the different cable elements and fibers location. For calculations we chose the fiber located closest to the cable surface to account for critical effects. The index difference between the core and cladding was kept constant, while the temperature changed. The coefficients values of the cable described are the following [13]:

$$
\begin{gathered}
\frac{d S_{0}}{d T}=-0.4 \cdot 10^{-6} \frac{\mathrm{ps}}{\mathrm{nm}^{2} \cdot \mathrm{km} \cdot \mathrm{K}}, \\
\frac{d \lambda_{0}}{d T}=0.026 \frac{\mathrm{nm}}{\mathrm{K}} .
\end{gathered}
$$

Equation (1) was solved for the chosen conditions and time delay was calculated. Since the time delay and temperature changes are linearly dependent, the signal delay in time can be found. Fig. 1 shows the dependence of the synchronization signal temporal delay induced by cable heating due to daily temperature oscillations As can be seen the temperature which induces the maximum tolerable time delay of $1.7 \mathrm{ps}$ accumulates every 2.3 hours. Therefore, for SCWQC systems an additional modulation signal adjustment must be performed to avoid the influence of the discussed dispersion factor.

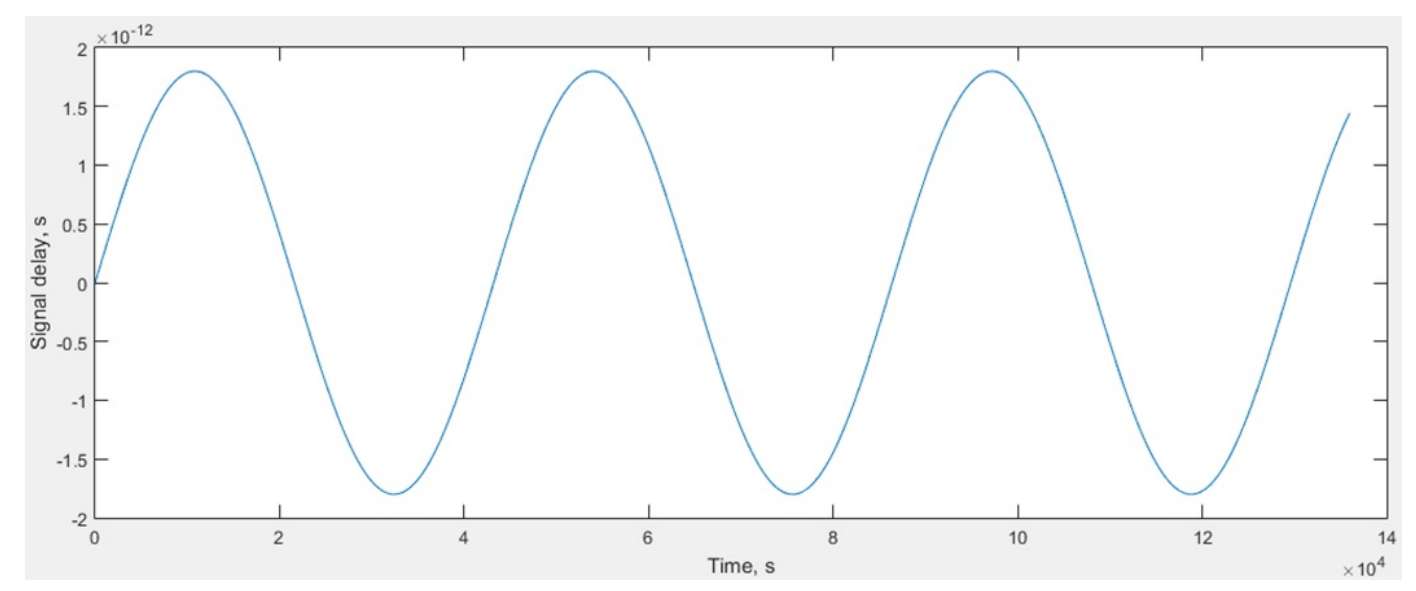

FIG. 1. Dependence of the signal delay time upon cable heating

\section{Conclusion}

We modeled the temperature dependence of the synchronization signal delay in optical fiber cables due to chromatic dispersion effects. The temperature dependence of zero dispersion wavelength and chromatic dispersion slope were considered. The temperature model was used to determine the necessary phase adjustment interval for the SCWQC synchronization system operating with a standard optical cable. It has been shown that the temperature effects cause the maximum tolerable signal delay of $1.7 \mathrm{ps}$ due to dispersion effects every 2.3 hours. Therefore, additional phase adjustment is required to compensate for this effect.

\section{Acknowledgements}

The research has been carried out with the financial support of the Government of the Russian Federation (074U01) and Ministry of Education and Science of the Russian Federation under grant agreement \#14.578.21.0112 (RFMEFI57815X0112) and contract No. 02.G25.31.0229. 


\section{References}

[1] Scarani V., Bechmann-Pasquinucci H., Cerf N.J., et al. The security of practical quantum key distribution. Reviews of Modern Physics, 2009, 81, P. 1302-1351.

[2] Bennett C., Brassard G. Quantum cryptography: Public key distribution and coin tossing. Proceedings of IEEE International Conference on Computers. Systems and Signal Processing, 1984, P. 175-179.

[3] Merolla J.M., Mazurenko Y., et al. Phase modulation transmission system for quantum cryptography. Optics letters, 1999, 24(2), P. 104106.

[4] Korzh B., Wen Lim C.C., et al. Provably secure and practical quantum key distribution over 307 km of optical fibre. Nature Photonics, 2015, 9, P. 163-168.

[5] Wang S., Chen W., et al. $2 \mathrm{GHz}$ clock quantum key distribution over $260 \mathrm{~km}$ of standard telecom fiber. Optics letters, 2012, 37(6), P. $1008-1010$.

[6] Kovalenko A.N. High-temperature superconductivity: From macro- to nanoscale structures. Nanosystems: Physics, Chemistry, Mathematics, 2016, 7(6), P. 941-970.

[7] Dubrovskaia V.D., Chivilikhin S.A. Temperature dependence of the optical fiber cable parameters in subcarrier wave quantum communication systems. Nanosystems: Physics, Chemistry, Mathematics, 2016, 7(2), P. 371-377.

[8] Gleim A.V., Egorov V.I., et al. Secure polarization-independent subcarrier quantum key distribution in optical fiber channel using BB84 protocol with a strong reference. Optics express, 2016, 24(3), P. 2619-2633.

[9] Gleim A., Egorov V., et al. Polarization Insensitive $100 \mathrm{MHz}$ Clock Subcarrier Quantum Key Distribution over a 45 dB Loss Optical Fiber Channel. CLEO: QELS_Fundamental Science. Optical Society of America, 2015, paper FF1A.5.

[10] Kutateladze S.S. Fundamentals of Heat Transfer, Moscow, Mashgiz Publishers, 1979, 417 p.

[11] Carslaw H.S., Jaeger J.C. Conduction of heat in solids. Clarendon Press, Oxford, 1959, 510 p.

[12] Kato T., Koyano Y., Nishimura M. Temperature dependence of chromatic dispersion in various types of optical fiber, Opt. Lett., 2000, 25, P. $1156-1158$.

[13] Hamp M.J., et al. Investigation into the temperature dependence of chromatic dispersion in optical fiber. IEEE Photonics Technology Letters, 14(11), P. 1524-1526. 